\title{
Entre sentido de pertenencia y potencial de desarrollo: la percepción del turismo en los residentes de Zaruma
}

Fecha de recepción: 2021-10-17 • Fecha de aceptación: 2021-12-14 • Fecha de publicación: 2022-02-10

Maritza Lisseth Cruz ${ }^{1}$

Universidad Técnica de Machala, Ecuador

mcruz3@utmachala.edu.ec

https://orcid.org/0000-0003-1759-8777

Lorenzo Bonisoli

Universidad Técnica de Machala, Ecuador

Ibonisoli@utmachala.edu.ec

https://orcid.org/0000-0003-3336-5658

Jose Andrés Albarracín ${ }^{3}$

Universidad Técnica de Machala, Ecuador

jaalbarracin_est@utmachala.edu.ec

https://orcid.org/0000-0001-9933-0883

\section{RESUMEN}

El turismo sostenible es un elemento potencial de desarrollo cultural y económico para una ciudad; sin embargo, para poder implementarlo es necesario el involucramiento de los residentes. El objetivo de este artículo es el análisis de aceptación del turismo sostenible en la ciudad de Zaruma, Ecuador. El modelo teórico utilizado se enfoca en dos factores: el potencial turístico y el sentimiento de pertenencia. Para el análisis se ha desarrollado una encuesta a 91 familias zarumeñas cuyas respuestas han sido elaboradas con la técnica SEM-PLS. Los resultados revelan como sí es cierto que el sentimiento de pertenencia a una comunidad juega un rol adverso a la percepción de la positividad de la actividad turística, los potenciales turísticos motivan con una fuerza mayor a los residentes hacia la aceptación de la actividad turística. 
PALABRAS CLAVE: marketing turístico, turismo sostenible, residentes, SEM-PLS

\section{ABSTRACT}

Sustainable tourism is a potential element of cultural and economic development for a city; however, in order to implement it, the involvement of residents is necessary. The objective of this article is to analyze the acceptance of sustainable tourism in the city of Zaruma, Ecuador. The theoretical model used focuses on two factors: tourism potential and sense of belonging. For the analysis, a survey of 91 families has been developed, whose answers have been elaborated with the SEM-PLS technique. The results reveal that while it is true that the feeling of belonging to a community plays an adverse role in the perception of the positivity of tourism activity, tourism potentials motivate residents to accept tourism activity with greater force.

KEYWORDS: tourism marketing, sustainable tourism, residents, SEM-PLS 


\section{Introducción}

Ecuador es un país que posee en su territorio una gran variedad de especies vegetales y animales endógenas, por esta razón es considerado como uno de los doce países de más alto nivel de biodiversidad del mundo. Además, el país es reconocido por sus atractivos turísticos culturales relacionados, sea a su pasado precolombino, o por su historia como colonia del Reino de España. Por esta razón, Ecuador es un país de alto interés turístico.

La actividad turística tiene como objetivo principal el desarrollo de la economía local, ya que permite el desprendimiento de varios factores positivos, como el aumento de las oportunidades de negocio, de las plazas de empleo (Aynalem et al., 2016), y de la calidad de las infraestructuras. Sin embargo, existen algunas consecuencias que afectan negativamente al destino turístico, como, por ejemplo, el posible aumento de la delincuencia, el incremento de los impuestos, la migración descontrolada, la elevación de la contaminación medioambiental y el desgaste de la identidad cultural, como de los atractivos naturales.

Frente a la problemática que se enfrenta, en los últimos años se han generado las prácticas de turismo sostenible (Ghobadi \& Verdian, 2016); es decir, el conjunto de prácticas que permiten, por una parte, el mantenimiento de los atractivos y de los servicios a los visitantes y, por otra parte, el respeto de las particularidades culturales de una sociedad y la reducción del impacto ambiental. Específicamente en el foro académico, el turismo sostenible se define como la actividad que, en su anhelo de satisfacer las necesidades de los turistas y de las empresas del sector, considera de forma primaria el impacto presente y futuro en el medioambiente, en la economía y en la sociedad. Según este enfoque, el uso de los recursos naturales es posible sólo mediante procesos que aseguren su conservación y las actividades de servicio al turista no pueden resultar en un perjuicio de los valores culturales.

Sin embargo, para poder implementar el turismo sostenible es necesario que los residentes estén involucrados en el desarrollo de las prácticas de sostenibilidad relacionadas a las actividades turísticas (Choi \& Murray, 2010). De hecho, el turismo sostenible no puede ser una práctica impuesta por las autoridades, porque de esta manera no resultaría en producir efecto beneficios y solo tendría el efecto de incomodar a la población residente. La única posibilidad para que se puedan implementar medidas de sostenibilidad es que los residentes acepten los sacrificios que estas prácticas podrían generar, porque entienden los beneficios que se producirían en el largo plazo y colaboren para el conseguimiento de dichos objetivos.

Zaruma, ciudad de la provincia ecuatoriana El Oro, fue denominada como patrimonio cultural de la humanidad ante la UNESCO por destacarse en la presencia de atractivos turísticos naturales y culturales extremadamente relevantes. Además, dentro del proyecto de ciudades mágicas de Ecuador, Zaruma fue identificada por el Ministerio del Turismo ecuatoriano como una de las 21 ciudades con esta calidad, por sus características históricas y naturales sobresalientes.

Sin embargo, la ciudad todavía no cuenta con un turismo masivo, más en cambio, con un turismo local, frecuentemente relacionado a la gastronomía y de estancia en promedio de un día (Villavicencio-Aguilar et al., 2018). Por ende, la ciudad cuenta con diversos atractivos que 
todavía no han sido explotados de la forma más eficiente y que podrían resultar en un aumento considerable del turismo del área.

Por lo consiguiente, el sentido de pertenencia es uno de los factores más importantes que influyen en esta decisión, ya que el punto de vista de los residentes es subjetivo, y cada uno va a tener un pensamiento distinto con respecto al bienestar de su ciudad. Considerando el fuerte potencial de desarrollo que representaría un aumento del turismo en esta ciudad, es necesario investigar la posible reacción de la población residente. En relación con esto, el objetivo de este artículo es el análisis de aceptación del turismo sostenible en la ciudad de Zaruma por parte de los ciudadanos zarumeños.

\subsection{Generalidades del turismo}

La actividad turística representa un potencial de desarrollo económico, ya que es razón de generación de oportunidad de negocios, de atracción de capital extranjero y de mejoramiento de infraestructuras (Aguilera, 2017).

Un destino turístico cuenta con atractivos únicos, ya sean naturales, históricos o culturales, que generan el interés en los potenciales turistas y que, por lo general, no son propiedad de una empresa en específico, más bien representan un bien común del cual se encarga la comunidad residente. Por ejemplo, en el caso del turismo rural, los turistas están atraídos por la cultura y costumbres, la gastronomía y los paisajes naturales. Alrededor de los atractivos, se desarrollan los servicios turísticos dirigidos a permitir y apoyar el turista en su experiencia, como, por ejemplo, el servicio de restauración, el hotelero y el de entretenimiento.

Una de las preocupaciones principales del marketing turístico es vincular las empresas turísticas a las características de los turistas que se diferencian en varias tipologías. El turista cultural está interesado en las formas culturales más formales de un destino, ya que prefiere los museos, las galerías de arte y las formas de entretenimiento cultural como los teatros y las manifestaciones. En general, es un turista de elevada capacidad de gasto y por ende elige los servicios hoteleros de alta calidad.

El turismo étnico es el interesado en conocer la historia de los lugares, nuevas culturas y diversos hábitos. Por lo general se adapta fácilmente al entorno y se deja llevar para vivenciar los hábitos de los residentes de la ciudad visitada. Busca los atractivos culturales típicos y únicos y tiende a evitar todo lo que no es típico de la ciudad visitada. Un tipo particular de turista cultural es el globetrotter, que se puede ver como un coleccionista de destinos visitados. Casi siempre auto organizado, privilegia los destinos lejanos de su propia residencia y de frecuentación inusual, este por lo general nunca visita dos veces el mismo destino (Miller, 2019).

Por su parte, el turista gastronómico está consciente que en cada ciudad la gastronomía es diferente, tiene su propia autenticidad y tiende a privilegiar este aspecto al respeto de los demás atractivos. Finalmente están el turista naturista, que busca un contacto especial con la naturaleza y prefiere los destinos más indicados para actividades de excursionismo, de exploración y en 
algunos casos de deportes extremos, finalmente el aventurero, que está interesado en vivir experiencias únicas.

Para que pueda crecer, el turismo necesita de la acción del marketing dirigido al turista. El marketing turístico, a causa de la específica naturaleza del turismo, tiene dos direcciones de aplicación: la primera se enfoca en la comunicación de la unicidad de los atractivos, mientras que la segunda en el desarrollo de los servicios turísticos. En el primer caso, el marketing turístico genera el concepto de "marca ciudad" (Gartner, 2014), tratando el nombre del destino con los mismos principios que se manejan las marcas de las empresas comerciales. La particularidad de la marca ciudad es que no existe un dueño exclusivo de la marca, en cambio, toda la comunidad es la beneficiaria del eventual éxito que la marca pueda lograr en el mercado. De consecuencia, cada negocio turístico particular participa en el resultado de la marca ciudad, ya que la calidad del servicio entregado no afecta exclusivamente una sola empresa, sino indirectamente a toda la comunidad. El marketing de los servicios turísticos tiene exactamente el objetivo de permitir que las acciones de cada negocio turístico sean relacionadas con la imagen de la marca ciudad en sí.

\subsection{Actitudes de los residentes}

Así como es importante que una localidad tenga una característica atractiva particular, también es fundamental que los residentes estén a favor de la actividad turística. Los recursos son elementos muy importantes para los residentes, la utilización de estos recursos depende únicamente de ellos, ya que deciden si compartir o no su cultura y sus hábitats naturales, con personas ajenas a su ciudad, ya que se pone en riesgo la pérdida o falta a sus costumbres (Chang et al., 2018; Wang, 2016). Además, es necesario que los residentes estén involucrados en la actividad, ya que de ellos depende tener una ciudad amigable y confortable para recibir turistas.

Los varios problemas relacionados al prejuicio ejercitado por las actividades humanas en el medio ambiente han generado la necesidad de que estas se acerquen a los requisitos de sostenibilidad. El desarrollo sostenible fue definido por como la exigencia de respetar las necesidades de las generaciones futuras en la satisfacción de las actuales. Aplicado al contexto turístico, el concepto de desarrollo sostenible conlleva toda una serie de acciones vueltas a la solución de problemas específicos.

El primero de ellos hace referencia a las consecuencias de la sobrepoblación provocada por los turistas genera en un destino. Un número significativo de turistas comporta un aumento en la producción de desechos, en el consumo energético, en la contaminación del aire y el desgaste de los atractivos naturales. Además, también desde el punto de vista social hay posibles consecuencias negativas de la actividad turística, como el posible aumento de criminalidad, la sobrepoblación en los servicios al ciudadano (transporte público, salud pública, etc.) y la generación de ruido.

El concepto de turismo sostenible quiere encontrar respuestas a los problemas antes mencionados, de forma que la actividad turística actual limite los impactos ambientales y sociales, 
y, por ende, que las generaciones futuras puedan aprovechar la oportunidad representada por el turismo.

\subsection{La ciudad de Zaruma}

El cantón de Zaruma se encuentra ubicado en la provincia de El Oro al sur del país, cuenta con muchos atributos, sus edificaciones históricas elaboradas con madera, artesanía y su exquisita gastronomía. Zaruma es una de las comunidades más antiguas de Ecuador, tiene aproximadamente 200 casas patrimoniales, construidas con técnicas antiguas. Así mismo es denominada como ciudad mágica, ofrece hermosos paisajes naturales como miradores, cascadas, lagos e incluso brinda lugares religiosos, entre estos está el santuario de la virgen del Carmen (Balcázar et al., 2019).

La UNESCO eligió a este cantón como patrimonio cultural de la humanidad, ya que Zaruma es una ciudad rica en atractivos y patrimonios ya antes mencionados, que la hacen una ciudad única, su clima es agradable, el cual permite a sus residentes y visitantes disfrutar de sus paisajes. Posee diez parroquias: una parroquia urbana y nueve parroquias rurales, todas destacando con una arquitectura neoclásica, su minería, su suelo y su producción de tierra permiten una economía sostenible para los habitantes del cantón, llegando a destacar productos como la caña de azúcar, panela y aguardiente, ganadería, productoras de leche, manjares, bocadillos y quesos. Su principal atracción internacional y nacional se ha convertido en "el café zarumeño". Zaruma, además de sus atractivos naturales y patrimoniales, goza de una riqueza aurífera, la misma que ha ayudado a la ciudad en su desarrollo económico.

\subsection{Soporte al turismo}

El turismo es una actividad con un fuerte potencial de desarrollo social; sin embargo, es necesario que los residentes acepten y sustenten esta actividad. Estudios especializados han mostrado como la resistencia de los residentes al desarrollo turístico ha limitado el avance de la localidad en términos de infraestructuras actualizadas y de servicios a la ciudadanía (Smith et al., 2021). Este constructo tiene el propósito de evaluar la aceptación del turismo por parte de los residentes, considerando la voluntad de estos últimos de compartir su cultura y su patrimonio cultural, que la ciudad atraiga más turistas, que se desarrollen nuevas atracciones turísticas y que se utilizaran los recursos económicos propios de la municipalidad para conseguir estos propósitos.

Una ciudad turística tiene grandes oportunidades de desarrollo económico, en término de emprendimiento y empleo. Además, tiene infraestructuras renovadas, lo cual hace que la ciudad se vea de interés para turistas. En la literatura se ha indicado como para los residentes los aspectos más importantes que el turismo puede incrementar es el flujo del dinero, relacionado a nuevas oportunidades de empleo y emprendimiento, el número y la eficiencia de las infraestructuras, la cantidad y calidad de eventos culturales y entretenimiento, y más en general del nivel de bienestar de las personas residentes (Jaafar et al., 2015).

Sin embargo, el turismo no es solo fomento de progreso y bienestar, sino que también conlleva posibles fenómenos negativos en la percepción de los residentes. Las situaciones que afectan 
negativamente una ciudad por el turismo, son de distinta naturaleza, pero, por lo general, el residente puede estar preocupado que la actividad turística no comporte un significativo incremento de nivel de vida y de oportunidades de trabajo, pero que solo aumente la presión humana poniendo en riesgo el patrimonio cultural de toda la comunidad (Ghahramani et al., 2020).

\section{Metodología}

\subsection{Hipótesis}

Los residentes apoyarán el turismo solo cuando las evaluaciones de las ventajas serán consideradas más importantes que las desventajas. De consecuencia se plantean las siguientes hipótesis:

H1: Los impactos positivos tienen una relación directa con el soporte al turismo de las personas residentes

\section{- Potencial turístico}

El atractivo en sí no es suficiente, aunque necesario, para que el turista sea motivado en visitar el destino. Por esta razón es necesario que este último también tenga servicios necesarios a los turistas, principalmente infraestructuras (Mamirkulova et al., 2020) y servicios de transporte. Los residentes que perciben que la ciudad tiene atractivos turísticos pueden considerar el impacto positivo del turismo. Por esta razón se plantea la siguiente hipótesis:

H2: los servicios turísticos son directamente relacionados al impacto positivo del turismo percibido por los residentes

\section{- Sostenibilidad ambiental}

Fomentar las acciones sustentables en la actividad turística garantiza, tanto a las generaciones presentes, como a las futuras, fuentes naturales y prósperas que pueden atraer siempre más turistas. Sin embargo, muchos residentes pueden percibir el riesgo que una actividad turística con un comportamiento irresponsable de los visitantes puede resultar en una alteración del estado del medio ambiente y el consecuente deterioro de los recursos naturales. Además, a parte de los comportamientos personales, el turismo implica un aumento de la presión humana en un lugar específico, que no siempre es bien tolerada por parte del ecosistema (Sari \& Nazli, 2020). Sin embargo, es posible pensar que los recursos naturales resultan ser identificados como atracciones únicas, y, por ende, motiven a crear infraestructura que permitan recibir a los turistas. Por esta razón se plantea la siguiente hipótesis:

H3: las actitudes de los residentes hacia la sostenibilidad ambiental son directamente relacionadas con los servicios turísticos 


\section{- Atracciones turísticas}

Un destino puede tener distintas atracciones turísticas, desde el punto de vista históricocultural, gastronómico o de la naturaleza. Las atracciones turísticas son específicas del destino y no pueden intercambiarse con otras. Estas pueden ser también la base para construir otras atracciones de segundo nivel, teóricamente menos importantes, pero conllevan a incrementar la experiencia del visitante (Gelbman, 2021). Un jardín zoológico o un parque de diversión pueden ser ejemplos emblemáticos de esta tipología de atracciones secundarias. De consecuencia, la primera condición para que haya interés por parte de los turistas en un destino es que existan atracciones únicas que no pueden ser disfrutadas en otro lugar. Siendo que tener atracciones es la condición necesaria para desarrollar la actividad turística, se plantea la siguiente hipótesis:

H4: las atracciones turísticas son directamente relacionada con los servicios turísticos

\section{- Sentimiento comunitario}

Ser parte de una comunidad no es solamente un dato demográfico, para muchas personas se traduce en un sentimiento de identidad (Yang \& Xin, 2016) y pertenencia a una entidad comunitaria. La influencia del grupo social en los individuos que lo forman es un elemento fundamental del estudio del comportamiento del consumidor. El sentimiento comunitario podría funcionar como un elemento motivador del turismo, ya que el destino sería más conocido y aumentaría la popularidad y respeto general; sin embargo, es más probable que el sentimiento de pertenencia a la comunidad genere la voluntad de proteger la comunidad de influencias externas que podrían poner en riesgo su integridad. Por esta razón se plantea la siguiente hipótesis:

H5: el sentimiento comunitario está inversamente relacionados con el impacto positivo del turismo, percibido por los residentes

\section{- Involucramiento comunitario}

Para que una persona se sienta miembro efectivo de un grupo es necesario que concretamente realice actividades en el grupo, ya que estas son evidencia del estatus de miembro (Khodyakov et al., 2013). Una pertenencia no acompañada por actividades reales sería percibida sólo como ficticia y no coagente para el individuo. En el caso de los residentes de un destino, una condición al sentido de pertenencia comunitaria se muestra en el involucramiento que el residente mantiene con la comunidad, desde el punto de vista del compartir información relevante sobre la comunidad, de tener conocimiento de los asuntos considerados más importantes y de realizar actividades comunitarias. De acuerdo a estas últimas consideraciones se plantea la siguiente hipótesis:

H6: el involucramiento comunitario es directamente relacionado con el sentimiento comunitario

\section{- Amor a la comunidad}

El involucramiento en una comunidad es posible si la persona ha desarrollado sentimiento de amor a la comunidad. Los motivos a la base del amor pueden ser los más variados y el análisis 
trasciende los objetivos de la presente investigación. En este contexto, es suficiente establecer la siguiente hipótesis:

H7: El amor a la comunidad está directamente relacionado con el involucramiento a la comunidad.

En la siguiente Figura 1 grafica lo comentado.

Figura 1.

Modelo teórico

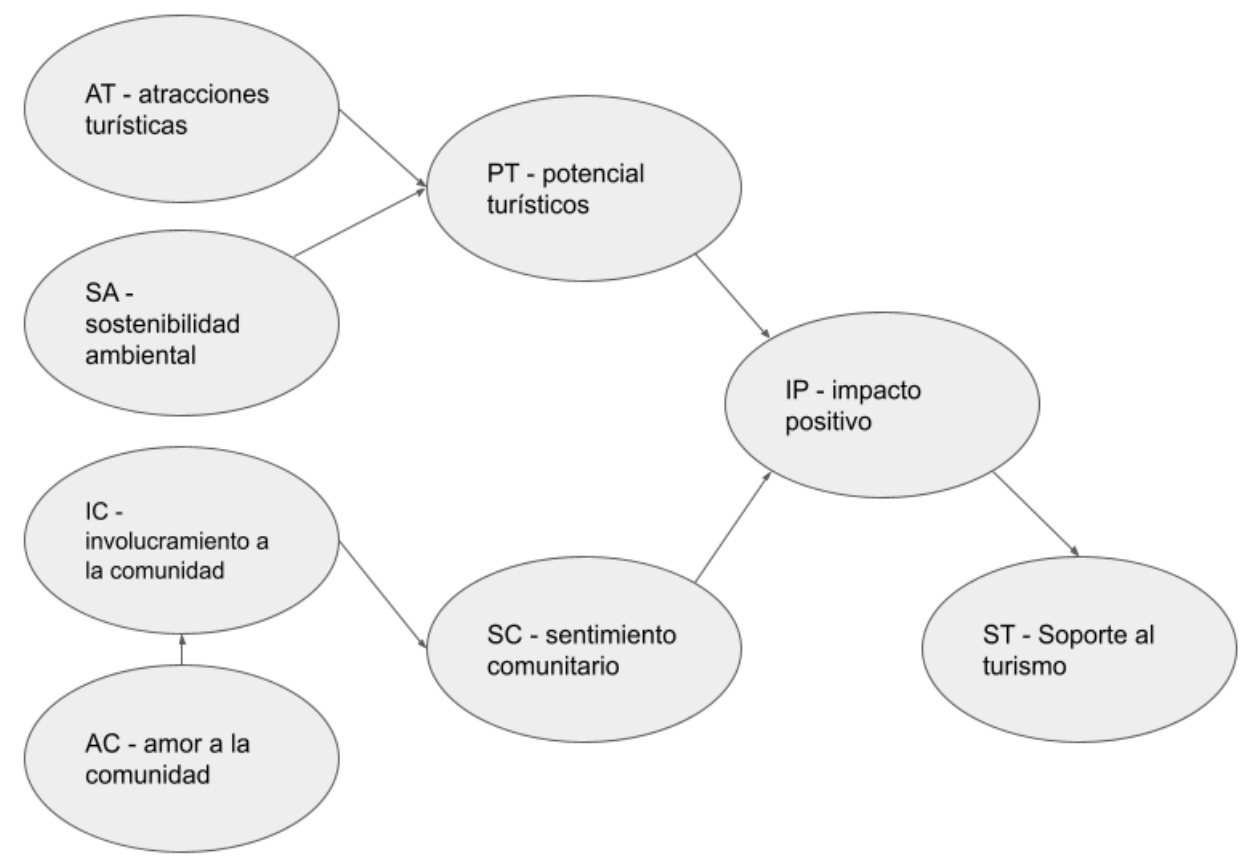

Esta investigación tiene el propósito de analizar la influencia de los servicios turísticos y del sentimiento comunitario en la percepción de los impactos del turismo y en el soporte al turismo de los residentes de la ciudad de Zaruma. Para realizar el análisis se ha desarrollado una encuesta compuesta por 19 ítems evaluados con escala likert de 5 puntos. El muestreo fue identificado según el concepto de conveniencia, que ha logrado aceptación en los estudios científicos, utilizando la técnica de bola de nieve. La encuesta fue enviada por e-mail a los residentes de la ciudad de Zaruma en los meses de junio y julio de 2021, logrando 91 respuestas. Los resultados han sido analizados con la técnica de SEM-PLS, elegida por su capacidad predictiva y de generar resultados con muestras pequeñas

\section{Resultados}

El análisis de los resultados ha sido conducido mediante un enfoque en dos etapas, en el cual la primera está representada por el análisis del modelo externo y la segunda por el análisis del modelo interno. 


\subsection{Análisis del modelo externo}

Este está dirigido a indagar la relación entre indicadores y variables mediante la evaluación de tres requisitos básicos del modelo: la fiabilidad interna, la validez convergente y la validez discriminante. La primera se refiere a la medida en la cual los indicadores de cada construcción están relacionados entre ellos. Los índices más frecuentemente usados en la literatura son la alfa de Cronbach y la fiabilidad compuesta, que en ambos casos deben tener valores entre 0.7 y 0.95 . La validez convergente se refiere en cambio a la medida en la cual cada indicador está relacionado a su propio constructo. Los índices utilizados en este caso son los de AVE y de cargas externas. La regla de oro indica en el primer caso valores mayores a 0.5 (Bagozzi \& Yi, 1988) y en el segundo mayores a 0.708 . Los resultados muestran que todos los índices mencionados respetan su respectiva norma de aceptación. De consecuencia, es posible afirmar que el modelo cumple con los supuestos de fiabilidad interna y validez convergente, ya que no solo los indicadores tienen una fuerte relación entre ellos, sino que tiene también una fuerte relación con su constructo.

La tercera evaluación es representada por la validez discriminante que evalúa que cada indicador no tenga una relación significativa con otros constructos del modelo. De esta manera se comprueba que cada constructo representa una realidad única del modelo y no determinada por otros constructos. Esta evaluación se realiza mediante tres análisis: los valores de las cargas cruzadas, el criterio de Fornell-Larcker y el indice HTMT. En el primer caso, los valores de las cargas cruzadas (generados cuando un indicador se relaciona con otro constructo) deben ser inferiores a los de las cargas externas (cuando un indicador se relaciona con su propio constructo). En el caso del criterio de Fornell-Larcker, las raíces cuadradas del AVE de cada constructo (valores en la diagonal) debe ser mayor a la correlación entre los diversos constructos (valores en la misma fila o columna). En el caso del índice HTMT, en la literatura se indica que los valores deben ser inferiores a 0.9 para que sea comprobada la validez discriminante. Los resultados muestran que todos los valores cumplen con los requisitos de aceptación respectivos, en las siguientes Tablas 2 , Tabla 3, Tabla 4 y Tabla 5 se representan.

Tabla 2.

Fiabilidad interna

\begin{tabular}{lcccc}
\hline & $\begin{array}{c}\text { Cronbach's } \\
\text { Alpha }\end{array}$ & rho_A & $\begin{array}{c}\text { Composite Relia- } \\
\text { bility }\end{array}$ & $\begin{array}{c}\text { Average Variance Extracted } \\
\text { (AVE) }\end{array}$ \\
\hline AC & 0,804 & 0,814 & 0,884 & 0,717 \\
\hline AT & 0,795 & 0,811 & 0,880 & 0,710 \\
\hline IC & 0,863 & 0,886 & 0,916 & 0,784 \\
\hline IP & 0,809 & 1.024 & 0,871 & 0,693 \\
\hline PT & 0,743 & 0,753 & 0,886 & 0,795 \\
\hline SA & 0,876 & 0,897 & 0,923 & 0,799 \\
\hline SC & 1.000 & 1.000 & 1.000 & 1.000 \\
\hline ST & 1.000 & 1.000 & 1.000 & 1.000 \\
\hline
\end{tabular}


Tabla 3.

Cargas externas y cruzadas

\begin{tabular}{ccccccccc}
\hline & AC & AT & IC & IP & PT & SA & SC & ST \\
\hline AC-1 & 0,839 & 0,196 & 0,208 & 0,390 & 0,498 & 0,431 & 0,079 & 0,382 \\
\hline AC-2 & 0,866 & 0,478 & 0,189 & 0,358 & 0,599 & 0,379 & 0,206 & 0,271 \\
\hline AC-3 & 0,834 & 0,682 & 0,251 & 0,239 & 0,391 & 0,226 & 0,251 & 0,269 \\
\hline AT-1 & 0,366 & 0,777 & 0,112 & 0,064 & 0,267 & 0,016 & 0,190 & 0,130 \\
\hline AT-2 & 0,541 & 0,846 & 0,124 & 0,123 & 0,290 & 0,176 & 0,199 & 0,358 \\
\hline AT-3 & 0,483 & 0,900 & 0,139 & 0,147 & 0,338 & 0,096 & 0,210 & 0,158 \\
\hline IC-1 & 0,258 & 0,153 & 0,865 & $-0,019$ & 0,105 & 0,101 & 0,461 & 0,015 \\
\hline IC-2 & 0,278 & 0,210 & 0,924 & $-0,040$ & 0,193 & 0,020 & 0,573 & 0,146 \\
\hline IC-3 & 0,134 & 0,003 & 0,865 & $-0,121$ & 0,036 & $-0,065$ & 0,452 & 0,120 \\
\hline IP-3 & 0,472 & 0,167 & $-0,065$ & 0,897 & 0,439 & 0,332 & $-0,140$ & 0,424 \\
\hline IP-4 & 0,207 & 0,053 & $-0,007$ & 0,830 & 0,221 & 0,217 & 0,040 & 0,246 \\
\hline IP-5 & 0,110 & 0,062 & $-0,081$ & 0,766 & 0,229 & 0,073 & $-0,118$ & 0,094 \\
\hline PT-1 & 0,549 & 0,326 & 0,158 & 0,374 & 0,908 & 0,374 & 0,216 & 0,165 \\
\hline PT-2 & 0,472 & 0,309 & 0,076 & 0,332 & 0,875 & 0,290 & 0,267 & 0,169 \\
\hline SA-1 & 0,353 & 0,142 & 0,034 & 0,234 & 0,318 & 0,879 & 0,094 & 0,162 \\
\hline SA-2 & 0,439 & 0,145 & 0,001 & 0,314 & 0,279 & 0,906 & $-0,022$ & 0,166 \\
\hline SA-3 & 0,299 & 0,043 & 0,028 & 0,236 & 0,391 & 0,897 & 0,094 & 0,193 \\
\hline SC-1 & 0,216 & 0,237 & 0,566 & $-0,107$ & 0,269 & 0,069 & 1.000 & 0,077 \\
\hline ST-2 & 0,362 & 0,254 & 0,108 & 0,359 & 0,187 & 0,196 & 0,077 & 1.000 \\
\hline & & & & & & & &
\end{tabular}

Tabla 4.

Fornell-Larcker

\begin{tabular}{ccccccccc}
\hline & AC & AT & IC & IP & PT & SA & SC & ST \\
\hline AC & 0,847 & & & & & & & \\
\hline AT & 0,552 & 0,843 & & & & & & \\
\hline IC & 0,259 & 0,149 & 0,885 & & & & & \\
\hline IP & 0,382 & 0,135 & $-0,064$ & 0,832 & & & & \\
\hline PT & 0,575 & 0,356 & 0,135 & 0,397 & 0,891 & & & \\
\hline SA & 0,398 & 0,116 & 0,025 & 0,288 & 0,376 & 0,894 & & \\
\hline SC & 0,216 & 0,237 & 0,566 & $-0,107$ & 0,269 & 0,069 & 1.000 & \\
\hline ST & 0,362 & 0,254 & 0,108 & 0,359 & 0,187 & 0,196 & 0,077 & 1.000 \\
\hline
\end{tabular}


Tabla 5.

HTMT

\begin{tabular}{|c|c|c|c|c|c|c|c|c|}
\hline & $A C$ & AT & IC & IP & PT & SA & SC & ST \\
\hline$A C$ & & & & & & & & \\
\hline AT & 0,665 & & & & & & & \\
\hline IC & 0,297 & 0,174 & & & & & & \\
\hline $\mathrm{IP}$ & 0,387 & 0,151 & 0,096 & & & & & \\
\hline $\mathrm{PT}$ & 0,753 & 0,461 & 0,163 & 0,449 & & & & \\
\hline SA & 0,495 & 0,167 & 0,090 & 0,294 & 0,452 & & & \\
\hline $\mathrm{SC}$ & 0,235 & 0,266 & 0,602 & 0,130 & 0,314 & 0,084 & & \\
\hline ST & 0,404 & 0,287 & 0,114 & 0,333 & 0,218 & 0,207 & 0,077 & \\
\hline
\end{tabular}

\subsection{Análisis del modelo interno}

El análisis del modelo interno se enfoca en la relación entre las variables. Los objetivos de este análisis son la verificación de que no existe colinealidad entre las variables, el valor del coeficiente de determinación $\mathrm{R}^{2}$, el cálculo del factor $\mathrm{f}^{2}$, las pruebas de las hipótesis del modelo y la evaluación del índice de predicción $\mathrm{Q}^{2}$.

La verificación de la colinealidad se ejecuta mediante el cálculo del valor VIF-interno, en la literatura se considera que este no debe superar el valor de 5 (Kock, 2015).

El coeficiente de determinación $\mathrm{R}^{2}$ se puede considerar como el propósito último de la técnica PLS, ya que representa la varianza de las variables endógenas explicada por el modelo. En la literatura se indican los valores de $\mathrm{R}^{2}$ de $0.25,0.50$ y 0.75 como débiles, moderados y fuertes, respectivamente. Los resultados muestran que los valores de $\mathrm{R}^{2}$ logrados por el modelo son bajos; sin embargo, considerando el tamaño reducido de la muestra, es posible aceptar valores de R2 hasta 0.1. La evaluación del coeficiente de determinación es generalmente acompañada del cálculo del factor $\mathrm{f}^{2}$ que expresa el aporte de $\mathrm{R}^{2}$ de una variable exógena en la variable endógena relacionada. La regla de oro identifica valores relevantes de $\mathrm{f}^{2}$ más altos de 0.02 y los resultados muestran que siempre se cumple este requisito. La prueba de las hipótesis del modelo se realiza a través de la técnica del Bootstrapping que genera los valores para contrastar la significancia de las hipótesis. Considerando un margen de significancia de 0.10 están aceptadas las hipótesis que muestran un p-valor menor a 0.1 . Los resultados muestran que todas las hipótesis del modelo son significativas.

Finalmente, el índice $\mathrm{Q}^{2}$ de Stone Geisser que se desarrolla mediante la técnica de Blindfolding indica la capacidad predictiva que el modelo genera en cada variable endógena. Los resultados muestran valores positivos de Q2 para cada variable, en las siguientes Tabla 6, 7, 8, 9 y 10 se muestra. 
Tabla 6.

VIF interno

\begin{tabular}{llllll}
\hline & IC & IP & PT & SC & ST \\
\hline AC & 1.000 & & & & \\
\hline AT & \multicolumn{5}{c}{1.014} \\
\hline IC & & & 1.000 & \\
\hline IP & & & \\
\hline PT & 1.078 & & \\
\hline SA & & 1.014 & \\
\hline SC & 1.078 & & \\
\hline ST & & & \\
\hline
\end{tabular}

Tabla 7.

$R^{2}$

\begin{tabular}{ccc}
\hline & $\mathbf{R}^{2}$ & $\mathbf{R}^{\mathbf{2}}$ Adjusted \\
\hline IC & 0,067 & 0,057 \\
\hline IP & 0,207 & 0,189 \\
\hline PT & 0,240 & 0,223 \\
\hline SC & 0,320 & 0,312 \\
\hline ST & 0,129 & 0,119 \\
\hline
\end{tabular}

Tabla 8.

$f^{2}$

\begin{tabular}{|c|c|c|c|c|c|}
\hline & IC & IP & PT & SC & ST \\
\hline$A C$ & 0,072 & & & & \\
\hline AT & & & 0,131 & & \\
\hline IC & & & & 0,470 & \\
\hline IP & & & & & 0,148 \\
\hline PT & & 0,247 & & & \\
\hline$S A$ & & & 0,149 & & \\
\hline SC & & 0,062 & & & \\
\hline ST & & & & & \\
\hline
\end{tabular}


Tabla 9.

BS

\begin{tabular}{|c|c|c|c|c|c|c|c|}
\hline & $\begin{array}{c}\text { Original } \\
\text { Sample (O) }\end{array}$ & $\begin{array}{c}\text { Sample } \\
\text { Mean (M) }\end{array}$ & $\begin{array}{l}\text { Standard Deviation } \\
\text { (STDEV) }\end{array}$ & $\begin{array}{l}\text { T Statistics } \\
(|\mathrm{O} / \mathrm{STDEV}|)\end{array}$ & $\begin{array}{c}\mathrm{P} \\
\text { Values }\end{array}$ & $5,00 \%$ & $95,00 \%$ \\
\hline $\begin{array}{c}\mathrm{AC} \rightarrow> \\
\text { IC }\end{array}$ & 0,259 & 0,270 & 0,083 & 3.107 & 0,002 & 0,133 & 0,413 \\
\hline $\begin{array}{c}\mathbf{A T} \rightarrow> \\
\mathbf{P T}\end{array}$ & 0,317 & 0,325 & 0,086 & 3.705 & 0,000 & 0,176 & 0,458 \\
\hline $\begin{array}{c}\text { IC -> } \\
\text { SC }\end{array}$ & 0,566 & 0,565 & 0,077 & 7.376 & 0,000 & 0,441 & 0,686 \\
\hline $\begin{array}{c}\text { IP }>> \\
\text { ST }\end{array}$ & 0,359 & 0,378 & 0,128 & 2.810 & 0,005 & 0,187 & 0,568 \\
\hline $\begin{array}{c}\text { PT } \rightarrow> \\
\text { IP }\end{array}$ & 0,459 & 0,466 & 0,100 & 4.580 & 0,000 & 0,294 & 0,632 \\
\hline $\begin{array}{c}\text { SA } \rightarrow> \\
\text { PT }\end{array}$ & 0,339 & 0,351 & 0,101 & 3.368 & 0,001 & 0,189 & 0,521 \\
\hline $\begin{array}{c}\mathrm{SC}-> \\
\text { IP }\end{array}$ & $-0,230$ & $-0,229$ & 0,073 & 3.153 & 0,002 & $-0,346$ & $-0,110$ \\
\hline
\end{tabular}

Tabla 10.

Q2

\begin{tabular}{cccc}
\hline SSO & SSE & $\begin{array}{c}\mathbf{Q}^{2} \text { (=1-SSE/ } \\
\text { SSO) }\end{array}$ \\
\hline AC & 273.000 & 273.000 & \\
\hline AT & 273.000 & 273.000 & \\
\hline IC & 273.000 & 260.589 & 0,045 \\
\hline IP & 273.000 & 244.713 & 0,104 \\
\hline PT & 182.000 & 152.913 & 0,160 \\
\hline SA & 273.000 & 273.000 & \\
\hline SC & 91.000 & 62.854 & 0,309 \\
\hline ST & 91.000 & 83.815 & 0,079 \\
\hline
\end{tabular}

\section{Conclusiones}

El objetivo de este trabajo fue encontrar la fuerza de la relación entre los potenciales turísticos, el sentimiento comunitario y la percepción de los impactos positivos del turismo en los residentes de la ciudad de Zaruma.

Los resultados revelan como sí es cierto que el sentimiento de pertenencia a una comunidad juega un rol adverso a la percepción de la positividad de la actividad turística, los potenciales turísticos motivan con una fuerza mayor a los residentes. Para próximas investigaciones podrían enfocarse en otros aspectos relacionados a la participación de los residentes en las actividades turísticas para identificar los principales motivadores de un desarrollo turístico en sentido sostenible. 


\section{Referencias}

Aguilera, A. V. (2017). Patrimonio, turismo y mercado inmobiliario en Tepoztlán, México. PASOS Revista de Turismo Y Patrimonio Cultural, 15(1), 181-193. https://doi.org/10.25145/j.pasos.2017.15.011

Aynalem, S., Birhanu, K., \& Tesefay, S. (2016). Employment Opportunities and Challenges in Tourism and Hospitality Sectors. Journal of Tourism \& Hospitality, 05(06). https://doi.org/10.4172/2167-0269.1000257

Bagozzi, R. P., \& Yi, Y. (1988). On the evaluation of structural equation models. Journal of the Academy of Marketing Science, 16(1), 74-94. https://doi.org/10.1007/BF02723327

Balcázar, P. E. L., Kirby, B. Z. R., \& Córdova, A. V. M. (2019). Contribución a las potencialidades de los destinos turísticos de Zaruma de la Provincia de El Oro. Gestión Ingenio y Sociedad, 3(2), 27-36.

Chang, K., Chien, H., Cheng, H., \& Chen, H.-I. (2018). The Impacts of Tourism Development in Rural Indigenous Destinations: An Investigation of the Local Residents' Perception Using Choice Modeling. Sustainability, 10(12), 4766. https://doi.org/10.3390/su10124766

Choi, H. C., \& Murray, I. (2010). Resident attitudes toward sustainable community tourism. Journal of Sustainable Tourism, 18(4), 575-594. https://doi.org/10.1080/09669580903524852

Gartner, W. C. (2014). Brand equity in a tourism destination. Place Branding and Public Diplomacy, 10(2), 108116. https://doi.org/10.1057/pb.2014.6

Gelbman, A. (2021). Tourist Experience and Innovative Hospitality Management in Different Cities. Sustainability, 13(12), 6578. https://doi.org/10.3390/su13126578

Ghahramani, L., McArdle, K., \& Fatorić, S. (2020). Minority Community Resilience and Cultural Heritage Preservation: A Case Study of the Gullah Geechee Community. Sustainability: Science Practice and Policy, 12(6), 2266. https://doi.org/10.3390/su12062266

Ghobadi, G. J., \& Verdian, M. S. (2016). The Environmental Effects of Tourism Development in Noushahr. Open Journal of Ecology, 06(09), 529-536. https://doi.org/10.4236/oje.2016.69050

Jaafar, M., Mostafa Rasoolimanesh, S., \& Lonik, K. A. T. (2015). Tourism growth and entrepreneurship: Empirical analysis of development of rural highlands. Tourism Management Perspectives, 14, 17-24. https://doi. org/10.1016/j.tmp.2015.02.001

Khodyakov, D., Stockdale, S., Jones, A., Mango, J., Jones, F., \& Lizaola, E. (2013). On Measuring Community Participation in Research. Health Education \& Behavior, 40(3), 346-354. https://doi. org/10.1177/1090198112459050 
Kock, N. (2015). Common method bias in PLS-SEM: A full collinearity assessment approach. International Journal of E-Collaboration, 11(4), 1-10. https://doi.org/10.4018/ijec.2015100101

Kourtit, K., Nijkamp, P., \& Östh, J. (2021). My home is my castle - Assessment of city love in Sweden. International Journal of Information Management, 58, 102213. https://doi.org/10.1016/j.ijinfomgt.2020.102213

Mamirkulova, G., Mi, J., Abbas, J., Mahmood, S., Mubeen, R., \& Ziapour, A. (2020). New Silk Road infrastructure opportunities in developing tourism environment for residents better quality of life. Global Ecology and Conservation, 24, e01194. https://doi.org/10.1016/j.gecco.2020.e01194

Miller, A. P. (2019). The Globetrotter: Cosmopolitan Travel, Connecting Cultures and Conjuring the 'Authentic' East, 1870-1920. [Tesis doctoral, University College London]. https://discovery.ucl.ac.uk/id/eprint/10076876

Sari, F. O., \& Nazli, M. (2020). Exploring the effects of "excessive tourism growth" on public health and ecosystem. Journal of Hospitality and Tourism Insights, 4(1), 1-17. https://doi.org/10.1108/JHTI-04-2020-0060

Smith, M. K., Sziva, I. P., \& Olt, G. (2021). Overtourism and Resident Resistance in Budapest. Travel and Tourism in the Age of Overtourism, 24-40. https://doi.org/10.4324/9781003140610-3

Villavicencio-Aguilar, C. E., Fernández-Espinosa, C. E., \& Jiménez-Idrovo, Í. V. (2018). Construcción de la identidad con base en el lenguaje. http://bdigital2.ula.ve:8080/xmlui/handle/654321/1740

Yang, Z., \& Xin, Z. (2016). Community Identity Increases Urban Residents' In-group Emergency Helping Intention. Journal of Community \& Applied Social Psychology, 26(6), 467-480. https://doi.org/10.1002/casp.2274 
Copyright (c) 2022 Maritza Lisseth Cruz, Lorenzo Bonisoli y Jose Andrés Albarracín

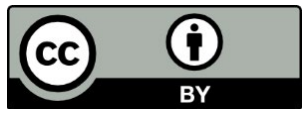

Este texto está protegido bajo una licencia internacional Creative Commons 4.0.

Usted es libre para Compartir-copiar y redistribuir el material en cualquier medio o formato - y Adaptar el documento - remezclar, transformar y crear a partir del material-para cualquier propósito, incluso para fines comerciales, siempre que cumpla las condiciones de Atribución. Usted debe dar crédito a la obra original de manera adecuada, proporcionar un enlace a la licencia, e indicar si se han realizado cambios. Puede hacerlo en cualquier forma razonable, pero no de forma tal que sugiera que tiene el apoyo del licenciante o lo recibe por el uso que hace de la obra.

\section{$\underline{\text { Resumen de licencia - Texto completo de la licencia }}$}

